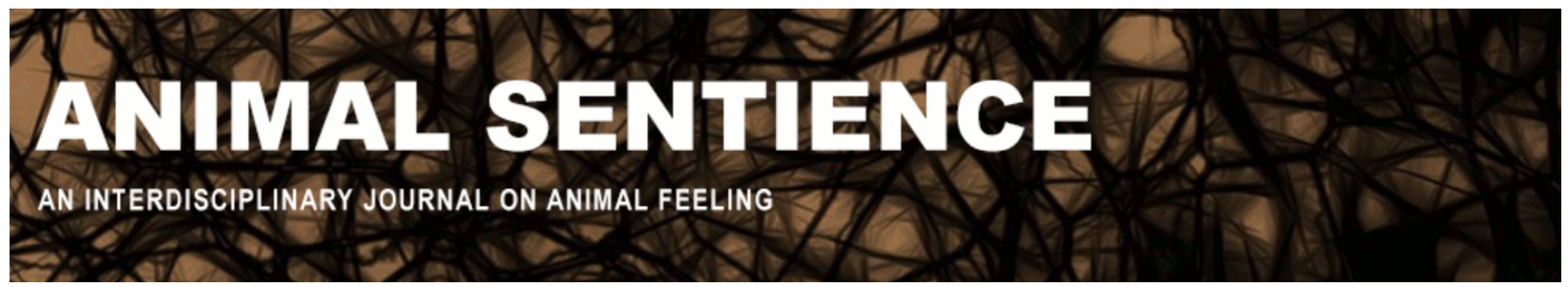

Mónus, Ferenc (2018) Competing activities as measures of fear and vigilance. Animal Sentience 15(8)

DOI: $10.51291 / 2377-7478.1312$

Date of submission: 2018-03-01

Date of acceptance: 2018-04-17

(c)

This article has appeared in the journal Animal

Sentience, a peer-reviewed journal on animal

cognition and feeling. It has been made open access,

free for all, by WellBeing International and deposited

in the WBI Studies Repository. For more information,

please contact

wbisr-info@wellbeingintl.org.

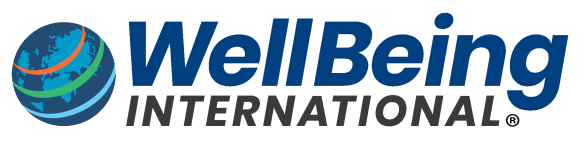

SOLUTIONS FOR PEOPLE, ANIMALS AND ENVIRONMENT 


\title{
Competing activities as measures of fear and vigilance
}

\author{
Commentary on Beauchamp on Fear \& Vigilance
}

\section{Ferenc Mónus}

Institute of Environmental Sciences

University of Nyíregyháza, Hungary

\begin{abstract}
In animal behavioural research on vigilance, visual signs of alertness are usually used to estimate perceived risk (an internal "fear" state) of free-ranging animals. Different measures of vigilance and competing activities (e.g., predator vigilance, conspecific vigilance, feeding, food handling) provide clues for better understanding vigilance behaviour. How efficiently does an animal in a vigilant/non-vigilant posture devote attention to threats or invest in other activities, such as searching for or handling food? Several species regularly withdraw to a sheltered spot when feeding in an abundant food patch, spending short periods in complete safety. Frequencies of feeding interruptions or false-alarm flights provide alternative measures of fear. I review how these phenomena may relate to the human understanding of the threats animals may perceive.
\end{abstract}

Ferenc Mónus is an evolutionary biologist studying foraging and antipredatory strategies in birds, the role of spatial position in behavioural responses of birds, human mate choice, and the methods and efficacy of environmental education.

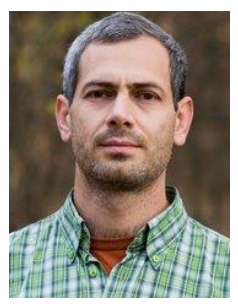
www.researchgate.net/profile/Ferenc_Monus

In the target article, Beauchamp (2017) provides a detailed discussion of how closely animal fear (a feeling) and vigilance (specific external signs of animals' alertness visually detectable by human observers) may be related. The measurement and function of vigilance were comprehensively reviewed by Beauchamp in 2015, but some important points may clarify the many facets of the widely used "vigilance" measurements. I suggest taking these points into consideration in assessing animal fear. I also draw attention to feeding interruptions and false-alarm flights as alternatives to vigilance for assessing animal fear.

\section{Measurement of Vigilance}

Alertness, an internal state in which animals are highly reactive in order to avoid some threat, frequently has external signs. Although we have only limited explicit knowledge of how closely external signs such as vigilance are related to the internal state (as reviewed by Beauchamp), the relationship between them is obvious and repeatedly demonstrated (e.g., Lima \& Bednekoff 1999, Harkin et al. 2000, Bednekoff \& Blumstein 2009). Studies usually measure alertness in species that display body or head postures readily interpreted as paying attention to a potential threat (i.e., predators or aggressive conspecifics). These postures, called vigilance behaviour or simply 
vigilance, include the upright body postures readily visible in meerkats (Suricata suricatta), several ground squirrel species, prairie dogs (Cynomys spp.), marmots (Marmota spp.) and susliks (Spermophilus spp.), as well as the head-up or scanning postures of birds and mammals (Quenette 1990, Treves 2000, Beauchamp 2015).

Since humans, including field researchers, perceive the world predominantly visually, the signs of alertness they use are mainly visual. On the other hand, again because researchers may observe species more easily in open landscapes than in visually obstructed ones (e.g., forests, water, etc.), the human-detected visual signs of alertness usually include erect postures for species living in open habitats. There have been much fewer studies of other signs, such as ear pointing, sniffing or even alertness to vibrations generated by potential enemies (Tobler \& Neuner-Jehle 1992, Hill 2009, Herberstein et al. 2014). These inter-related auditory, olfactory or vibrotactile signs of alertness make it difficult to measure animal fear based on only a single external sign.

In the case of vigilance behaviour, also called scanning or monitoring behaviour, we already have many studies on several species (recently reviewed in Beauchamp 2015). The tendency for vigilance to decrease when safe cover is nearer (Barta et al. 2004, Mónus \& Barta 2011), flock size increases (Barta et al. 2004, Mónus \& Barta 2011), social information on threats is more available (Bekoff 1995, Fernández-Juricic et al. 2005), or competitors are not threatening (Beauchamp 2016) illustrates the functions of vigilance behaviour. On the other hand, the better our understanding of the function of vigilance behaviour, the more complex and the more questions arise. Which aspect of the vigilance behaviour represents valuable information in a specific case? We can measure the proportion of time in the alert stance during the whole observation, the frequency of scans (or interscan intervals), and the length of the individual scans. As part of their antipredator strategy, animals may adjust each aspect separately (Beauchamp 2015). Which aspect is more important under any particular condition has received little attention (Bednekoff \& Lima 2002, Sirot \& Pays 2011, Beauchamp \& Ruxton 2016). A meta-analysis of the studies measuring different aspects of vigilance behaviour would be very useful in providing guidelines.

\section{Compatibility between Vigilance and Other Activities}

Animals must consider several conflicting factors. Vigilance may be costly if the time allocated for it would also be important for other activities, most frequently foraging time. Selection favours those behaviours or abilities that allow animals to adopt two conflicting activities. For instance, head-down food searching may allow some predator detection in rats (Wallace et al. 2013) and in several birds (Lima \& Bednekoff 1999, Fernández-Juricic et al. 2008), and also some detection of social information (Fernández-Juricic et al. 2005). Strategic adjustment of behaviour to internal and external conditions may result in different vigilance patterns despite having the same level of internal fear. Compatibility is known to be only partial (i.e., one of the two or both compatible activities being less efficient than devoting behaviour entirely to one purpose), complicating the link between vigilance and fear.

Several recent studies address the different types of vigilance and their compatibility (Fernández-Juricic 2012). Low-cost (or routine) and high-cost vigilance are frequently distinguished relative to the costs of foraging (e.g., Baker et al. 2011, Favreau et al. 2013, 2015). 
In several cases, low-cost vigilance is devoted mainly to social contexts (e.g., Coolen \& Giraldeau 2003, Monclús \& Rödel 2008) while high-cost vigilance is devoted mainly to antipredator contexts (e.g., Coolen \& Giraldeau 2003, Monclús \& Rödel 2008, Favreau et al. 2013). Some studies also consider gaze orientation (acute vision) to fine-tune the distinction between lower-cost social vigilance (Butler et al. 2016) and higher-cost antipredator vigilance (Butler \& Fernández-Juricic 2018). Even short open-eyed periods during sleep allow visual alertness in incubating birds (Javůrková et al. 2011). Tiny details can count.

\section{Feeding Interruptions and False-Alarm Flights as Estimates of Fear}

If the link between vigilance and fear is frequently decoupled, can we find any measures that may better characterize fear? Flight-initiation distance (distance from a simulated or real predator that makes animals escape) is frequently measured in several species (Stankowich \& Blumstein 2005, pp. 174-176 in Beauchamp 2015). Although this could be one possible candidate for measuring

fear, escape decisions are, like vigilance, immensely complex in animals. Influencing factors include predator type, prey condition, group size and composition, food abundance, habitat (vegetation) type, refuge distance, as well as prey personality (shyness or boldness), individual variability and habituation (reviewed in Cooper \& Blumstein 2015).

A much more promising though much less studied candidate would be feeding interruptions and false-alarm flights. Frequent feeding interruptions occur in many species when exploiting abundant food patches (e.g., Lima et al. 1985), typical at bird feeders (Barnard 1980, Lendrem 1983). More feeding interruptions may result from more fear (Lendrem 1983, Randler 2006, Wheeler \& Hilk 2014). Tree sparrow flocks have much shorter but more frequent feeding bouts under riskier conditions (Mónus \& Barta 2011). Contrary to expectations, with more feeding bouts, the time lags between two successive feeding bouts were longer in the riskier condition (see Table 1 in Mónus \& Barta 2011 for more details). This also underlies the role of perceived predation-risk in the time-budget pattern we observed (Mónus et al. 2016). In that case, feeding interruptions contributed to the anti-predator strategy of the sparrows; however, misclassifications of social information on threats (i.e., false alarms) are also common and result in feeding interruptions (Lima 1994, Cresswell et al. 2000, Beauchamp \& Ruxton 2007). When foragers perceive greater risk or fear, false alarms may also increase. Both feeding interruptions and false-alarm flights should be further investigated in relation to perceived risk and animal fear.

\section{Concluding Remarks}

Although investigating animal vigilance has been a long tradition in behavioural ecology, several details remain to scrutinize in future studies, such as the function of inter-scan intervals, scan length, and low- and high-cost forms of vigilance. Further experiments are also needed on the relationship of feeding interruptions to fear and perceived risk: are they an anti-predator strategy or false alarms? 


\section{References}

Baker, D. J., Stillman, R. A., Smart, S. L., Bullock, J. M., \& Norris, K. J. (2011). Are the costs of routine vigilance avoided by granivorous foragers?. Functional Ecology, 25(3), 617-627.

Barnard, C. J. (1980). Flock feeding and time budgets in the house sparrow (Passer domesticus L.). Animal Behaviour, 28(1), 295-309.

Barta, Z., Liker, A., \& Mónus, F. (2004). The effects of predation risk on the use of social foraging tactics. Animal Behaviour, 67(2), 301-308.

Beauchamp, G. (2015). Animal vigilance: Monitoring predators and competitors. Academic Press.

Beauchamp, G. (2016). Function and structure of vigilance in a gregarious species exposed to threats from predators and conspecifics. Animal Behaviour, 116, 195-201.

Beauchamp, G. (2017). What can vigilance tell us about fear?. Animal Sentience 15(1).

Beauchamp, G., \& Ruxton, G. D. (2007). False alarms and the evolution of antipredator vigilance. Animal Behaviour, 74(5), 1199-1206.

Beauchamp, G., \& Ruxton, G. D. (2016). Modeling scan and interscan durations in antipredator vigilance. Journal of Theoretical Biology, 390, 86-96.

Bednekoff, P. A., \& Blumstein, D. T. (2009). Peripheral obstructions influence marmot vigilance: Integrating observational and experimental results. Behavioral Ecology, 20(5), 1111-1117.

Bednekoff, P. A., \& Lima, S. L. (2002). Why are scanning patterns so variable? An overlooked question in the study of anti-predator vigilance. Journal of Avian Biology, 33(2), 143-149.

Bekoff, M. (1995). Vigilance, flock size, and flock geometry: Information gathering by western evening grosbeaks (Aves: Fringillidae). Ethology, 99, 150-161.

Butler, S. R., \& Fernández-Juricic, E. (2018). European starlings use their acute vision to check on feline predators but not on conspecifics. PloS One, 13(1), e0188857.

Butler, S. R., Hosinski, E. C., Lucas, J. R., \& Fernández-Juricic, E. (2016). Social birds copy each other's lateral scans while monitoring group mates with low-acuity vision. Animal Behaviour, 121, 21-31.

Coolen, I., \& Giraldeau, L. A. (2003). Incompatibility between antipredatory vigilance and scrounger tactic in nutmeg mannikins, Lonchura punctulata. Animal Behaviour, 66(4), 657-664.

Cooper, Jr., W. E., \& Blumstein, D. T. (Eds.). (2015). Escaping from predators: An integrative view of escape decisions. Cambridge University Press.

Cresswell, W., Hilton, G. M., \& Ruxton, G. D. (2000). Evidence for a rule governing the avoidance of superfluous escape flights. Proceedings of the Royal Society of London B: Biological Sciences, 267(1444), 733-737.

Favreau, F. R., Pays, O., Fritz, H., Goulard, M., Best, E. C., \& Goldizen, A. W. (2015). Predators, food and social context shape the types of vigilance exhibited by kangaroos. Animal Behaviour, 99, 109-121.

Favreau, F. R., Pays, O., Goldizen, A. W., \& Fritz, H. (2013). Short-term behavioural responses of impalas in simulated antipredator and social contexts. PloS One, 8(12), e84970.

Fernández-Juricic, E. (2012). Sensory basis of vigilance behavior in birds: Synthesis and future prospects. Behavioural Processes, 89, 143-152.

Fernández-Juricic, E., Gall, M. D., Dolan, T., Tisdale, V., \& Martin, G. R. (2008). The visual fields of two ground-foraging birds, House Finches and House Sparrows, allow for simultaneous foraging and antipredator vigilance. Ibis, 150(4), 779-787.

Fernández-Juricic, E., Smith, R., \& Kacelnik, A. (2005). Increasing the costs of conspecific scanning in socially foraging starlings affects vigilance and foraging behaviour. Animal Behaviour, 69(1), 73-81.

Harkin, E. L., van Dongen, W. F., Herberstein, M. E., \& Elgar, M. A. (2000). The influence of visual obstructions on the vigilance and escape behaviour of house sparrows, Passer domesticus. Australian Journal of Zoology, 48(3), 259-263. 
Herberstein, M. E., Wignall, A. E., Hebets, E. A., \& Schneider, J. M. (2014). Dangerous mating systems: Signal complexity, signal content and neural capacity in spiders. Neuroscience \& Biobehavioral Reviews, 46, 509-518.

Hill, P. S. M. (2009). How do animals use substrate-borne vibrations as an information source? Naturwissenschaften, 96, 1355-1371.

Javůrková, V., Hořák, D., Kreisinger, J., Klvaňa, P., \& Albrecht, T. (2011). Factors affecting sleep/vigilance behaviour in incubating mallards. Ethology, 117(4), 345-355.

Lendrem, D. W. (1983). Predation risk and vigilance in the blue tit (Parus caeruleus). Behavioral Ecology and Sociobiology, 14(1), 9-13.

Lima, S. L. (1994). Collective detection of predatory attack by birds in the absence of alarm signals. Journal of Avian Biology, 25(4), 319-326.

Lima, S. L., \& Bednekoff, P. A. (1999). Back to the basics of antipredatory vigilance: Can nonvigilant animals detect attack?. Animal Behaviour, 58(3), 537-543.

Lima, S. L., Valone, T. J., \& Caraco, T. (1985). Foraging-efficiency-predation-risk trade-off in the grey squirrel. Animal Behaviour, 33(1), 155-165.

Monclús, R., \& Rödel, H. G. (2008). Different forms of vigilance in response to the presence of predators and conspecifics in a group-living mammal, the European Rabbit. Ethology, 114(3), 287-297.

Mónus, F., \& Barta, Z. (2011). Degree of synchronization of tree sparrows in flocks under different predation risk. Behaviour, 148(5), 733-744.

Mónus, F., \& Barta, Z. (2016). Is foraging time limited during winter?-A feeding experiment with Tree Sparrows under different predation risk. Ethology, 122(1), 20-29.

Quenette, P. Y. (1990). Functions of vigilance behaviour in mammals: A review. Acta Oecologica, 11(6), 801-818.

Randler, C. (2006). Red squirrels (Sciurus vulgaris) respond to alarm calls of Eurasian jays (Garrulus glandarius). Ethology, 112(4), 411-416.

Sirot, E., \& Pays, O. (2011). On the dynamics of predation risk perception for a vigilant forager. Journal of Theoretical Biology, 276(1), 1-7.

Stankowich, T., \& Blumstein, D. T. (2005). Fear in animals: A meta-analysis and review of risk assessment. Proceedings of the Royal Society of London B: Biological Sciences, 272(1581), 2627-2634.

Tobler, I., \& Neuner-Jehle, M. (1992). 24-h variation of vigilance in the cockroach Blaberus giganteus. Journal of Sleep Research, 1(4), 231-239.

Treves, A. (2000). Theory and method in studies of vigilance and aggregation. Animal Behaviour, 60(6), 711-722.

Wallace, D. J., Greenberg, D. S., Sawinski, J., Rulla, S., Notaro, G., \& Kerr, J. N. (2013). Rats maintain an overhead binocular field at the expense of constant fusion. Nature, 498(7452), 65.

Wheeler, H. C., \& Hik, D. S. (2014). Giving-up densities and foraging behaviour indicate possible effects of shrub encroachment on arctic ground squirrels. Animal Behaviour, 95, 1-8. 